\title{
Early gastric cancer management
}

\author{
A. Hussain · T. Singhal - A. Biju • H. Almusawy • \\ P. Sinha
}

Published online: 25 December 2009

(C) Springer Science+Business Media, LLC 2009

We read with great interest the article by Filippo Catalano and colleagues regarding early gastric cancer management [1].We appreciate the authors' efforts to manage their series using a unique minimal access approach. The results were encouraging, especially for the management of cardia lesions, which are technically challenging. However, we have some points that we consider complementary to the educational value of the study.

First, the curative resection was $56 \%$ in the endoscopic mucosal resection (EMR) group compared with $92 \%$ in the endoscopic submucosal dissection (ESD) group. The authors allowed a 5-mm safe margin for their resections. If a $1-\mathrm{cm}$ margin is used, the percentage of curative resection could be better.

Second, we find it important to know the results of preoperative investigations (e.g., endoscopic ultrasound) to determine which patients are to be included in the study.

Finally, in about $50 \%$ of the cases, the tumor size was $5-10 \mathrm{~mm}$. Such sizes can be missed during routine

gastroscopy, and we question whether indigo carmine should be used for all high-risk patients instead of the patients who would need ESD [2].

We appreciate a kind response from the authors.

Disclosures Abdulzahra Hussain, Tarun Singhal, Aravind Biju, Hussein Almusawy, and Prakash Sinha have no conflicts of interest or financial ties to disclose.

\section{References}

1. Catalano F, Trecca A, Rodella L, Lombardo F, Tomezzoli A, Battista S, Silano M, Gaj F, de Manzoni G (2009) The modern treatment of early gastric cancer: our experience in an Italian cohort. Surg Endosc 23:1581-1586

2. Iizuka T, Kikuchi D, Hoteya S, Yahagi N (2008) The acetic acid + indigo carmine method in the delineation of gastric cancer. J Gastroenterol Hepatol 23:1358-1361

A. Hussain $(\bowtie) \cdot$ T. Singhal $\cdot$ A. Biju $\cdot$ H. Almusawy $\cdot$ 